\section{(6) OPEN ACCESS}

\title{
Mortality and morbidity after high-dose methylprednisolone treatment in patients with acute cervical spinal cord injury: a propensity-matched analysis using a nationwide administrative database
}

\author{
Hirotaka Chikuda, ${ }^{1}$ Hideo Yasunaga, ${ }^{2}$ Katsushi Takeshita, ${ }^{1}$ Hiromasa Horiguchi, ${ }^{2}$ \\ Hiroshi Kawaguchi, ${ }^{1}$ Kazuhiko Ohe, ${ }^{3}$ Kiyohide Fushimi, ${ }^{4}$ Sakae Tanaka ${ }^{1}$
}

\begin{abstract}
Department of Orthopaedic Surgery, Faculty of Medicine The University of Tokyo, Tokyo, Japan ${ }^{2}$ Department of Health Management and Policy, Graduate School of Medicine, The University of Tokyo, Tokyo, Japan

${ }^{3}$ Department of Medical Informatics and Economics, Graduate School of Medicine, The University of Tokyo, Tokyo, Japan

${ }^{4}$ Department of Health Care Informatics, Tokyo Medical and Dental University, Tokyo, Japan

\section{Correspondence to} Dr Hirotaka Chikuda, Department of Orthopaedic Surgery, Faculty of Medicine, The University of Tokyo, 7-3-1 Hongo, Bunkyo-ku, Tokyo 113-8655, Japan; chikuda-tky@umin.ac.jp
\end{abstract}

Received 8 October 2012 Revised 18 December 2012 Accepted 19 December 2012 Published Online First 28 February 2013

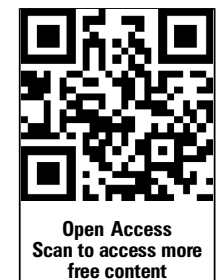

\footnotetext{
To cite: Chikuda $\mathrm{H}$ Yasunaga $\mathrm{H}$, Takeshita $\mathrm{K}$ et al. Emerg Med J 2014;31:201-206.
}

\begin{abstract}
Objective To examine the magnitude of the adverse impact of high-dose methylprednisolone treatment in patients with acute cervical spinal cord injury $(\mathrm{SCl})$.

Methods We examined the abstracted data from the Japanese Diagnosis Procedure Combination database, and included patients with ICD-10 code S141 who were admitted on an emergency basis between 1 July and 31 December in 2007-2009. The investigation evaluated the patients' sex, age, comorbidities, Japan Coma Scale, hospital volume and the amount of methylprednisolone administered. One-to-one propensity-score matching between high-dose methylprednisolone group (>5000 mg) and control group was performed to compare the rates of in-hospital death and major complications (sepsis; pneumonia; urinary tract infection; gastrointestinal ulcer/bleeding; and pulmonary embolism).
\end{abstract}

Results We identified 3508 cervical SCI patients (2652 men and 856 women; mean age, $60.8 \pm 18.7$ years) including 824 (23.5\%) patients who received high-dose methylprednisolone. A propensity-matched analysis with 824 pairs of patients showed a significant increase in the occurrence of gastrointestinal ulcer/bleeding $(68 / 812$ vs $31 / 812 ; p<0.001)$ in the high-dose methylprednisolone group. Overall, the high-dose methylprednisolone group demonstrated a significantly higher risk of complications (144/812 vs 96/812;OR, $1.66 ; 95 \% \mathrm{Cl} 1.23$ to $2.24 ; p=0.001)$ than the control group. There was no significant difference in in-hospital mortality between the high-dose methylprednisolone group and the control group ( $p=0.884)$.

Conclusions Patients receiving high-dose methylprednisolone had a significantly increased risk of major complications, in particular, gastrointestinal ulcer/bleeding. However, high-dose methylprednisolone treatment was not associated with any increase in mortality.

\section{INTRODUCTION}

Methylprednisolone is one of the most investigated agents for its neuroprotective potential, and remains the only drug used worldwide for acute spinal cord injury (SCI). The beneficial effect of high-dose methylprednisolone was initially reported in a series of National Acute Spinal Cord Injury Studies (NASCIS) in the 1990s. ${ }^{1}{ }^{2}$ Specifically, NASCIS-2 compared $24 \mathrm{~h}$ of high-dose methylprednisolone (given as a bolus of $30 \mathrm{mg} / \mathrm{kg}$ over $15 \mathrm{~min}$ followed by a continuous infusion of $5.4 \mathrm{mg} / \mathrm{kg} / \mathrm{h}$ ) with placebo in acute SCI patients. ${ }^{1}$ Patients receiving methylprednisolone within $8 \mathrm{~h}$ of injury were reported to have greater neurologic improvement at 6 months. Results of NASCIS-3 further indicated slightly more recovery following $48 \mathrm{~h}$ of treatment than after $24 \mathrm{h.}^{2}$ Following publication of the NASCIS trials, the regimen of these trials was rapidly adopted worldwide; however, subsequent debate over the efficacy and safety of high-dose methylprednisolone treatment ${ }^{3-5}$ has led to serious differences of opinion in the medical community, and considerable variations in current practice. ${ }^{6-9}$

According to a recent Cochrane review, ${ }^{10}$ NASCIS-2 showed a weak trend towards an increase in complications, including wound infection (OR 2.11; 95\% CI 0.81 to 5.49 ) and gastrointestinal haemorrhage (OR 1.48; 95\% CI 0.48 to 4.56). The high-dose methylprednisolone group showed slightly lower 180 -day mortality than the control group (7/162 vs $12 / 171$; OR $0.6295 \%$ CI 0.25 to 1.53$)$. On the other hand, NASCIS-3, comparing $24 \mathrm{~h}$ and $48 \mathrm{~h}$ methylprednisolone administration, found a trend towards increased rates of severe pneumonia (OR 2.25; 95\% CI 0.71 to 7.15 ) and sepsis (OR 4.00; 95\% CI 0.45 to 35.38 ) in the $48 \mathrm{~h}$ treatment group. Mortality was not significantly different between the two groups.

Although many studies following the NASCIS trials reported a trend toward increased complications after high-dose methylprednisolone treatment, ${ }^{11-15}$ the magnitude of its negative impact remains unclear. The reported incidence of complications after high-dose methylprednisolone administration varied greatly between studies, primarily because of small sample sizes and bias in selection of the study population. In addition, it is unknown whether high-dose methylprednisolone negatively affects the survival of SCI patients. Despite widespread use of this treatment, information from highlevel evidence about the risks associated with highdose methylprednisolone administration is lacking. We therefore conducted a retrospective observational study based on a propensity score-matched analysis of data from a nationwide administrative database to examine the risk of high-dose methylprednisolone treatment after acute cervical SCI.

\section{METHODS}

\section{Diagnosis Procedure Combination database}

The Diagnosis Procedure Combination (DPC) is a case-mix patient classification system which was 
launched in 2002 by the Ministry of Health, Labour and Welfare of Japan, and was linked with a lump-sum payment system. ${ }^{16}$ All 82 university teaching hospitals are obliged to adopt this system, but adoption by community hospitals is voluntary. The survey in the participating hospitals is conducted between 1 July and 31 December each year by the DPC research group, in collaboration with the Ministry of Health, Labour and Welfare. In 2009, the number of participating hospitals was 818 and the number of patients included was 2.57 million, which represented approximately $40 \%$ of all inpatient admissions to acute care hospitals in Japan. The database includes administrative claims data and the following data: unique identifiers of hospitals; patient age and sex; diagnoses, comorbidities at admission and complications after admission recorded with text data in the Japanese language and the International Classification of Diseases, 10th Revision (ICD-10) codes; consciousness level at admission measured with the Japan Coma Scale (JCS; see Appendix); discharge status; and drugs administered. ${ }^{17}$ In the DPC database, complications that occur after admission are clearly differentiated from comorbidities that already present at admission. To optimise the accuracy of the recorded diagnoses, physicians in charge are obliged to record the diagnoses with reference to medical charts. Because of the anonymous nature of the data, informed consent was waived when this study was approved by the institutional review board at The University of Tokyo.

\section{Patient selection and data}

Using the DPC database, we identified patients who had an emergency admission to the participating hospitals with a diagnosis of cervical SCI (ICD-10 code, S141) between July and December, 2007-2009. Patients who were transferred from other hospitals were excluded. Although we were unable to confirm the presence of a neurological deficit in each patient, miscoding is relatively unlikely because the DPC data are coded by physicians and subjected to an audit. The list of drugs used during hospitalisation was reviewed for each patient, and we identified patients who started high-dose methylprednisolone treatment for acute cervical SCI at admission and received a total of $\geq 5000 \mathrm{mg}$ methylprednisolone infusion. In Japan, many elderly patients who sustain a cervical SCI are lean. For a $40 \mathrm{~kg}$ person, the total dosage amounted to $6168 \mathrm{mg}$ in the NASCIS-2 protocol. Therefore, we set a cut-off value of $5000 \mathrm{mg}$. As a control group, we identified cervical SCI patients who did not receive methylprednisolone, or those who received less than $500 \mathrm{mg}$ methylprednisolone during hospitalisation. We selected this cut-off value according to the definition of 'highdose' adopted by Sauerland et al $^{18}$ (>15 mg/kg (600 mg for a $40 \mathrm{~kg}$ person) or $>1000 \mathrm{mg}$ ).

We assessed patient background, including age, sex, JCS score and Charlson Comorbidity Index (CCI). JCS 0 indicates patients with alert consciousness; JCS one-digit codes (1-3) indicate patients who are drowsy but awake without any stimuli; JCS two-digit codes (10-30) indicate patients with somnolence who can be aroused with some stimuli; JCS three-digit codes (100300) indicate coma. ${ }^{19}$ The JCS and the Glasgow Coma Scale assessments are well correlated. The CCI is a prognostic index as a means for quantifying the prognosis of patients enrolled in a large cohort, and is used widely to measure the case-mix with administrative data. This index is based on a point scoring system (from 0 to 40 ) for the presence of specific associated diseases. Quan et $a l^{20}$ provided a validated chart showing how each comorbidity corresponds to a set of ICD-10 codes. $^{20}$ Based on Quan's protocol, each ICD-10 code of comorbidity was converted into a score, and was summed for each patient to determine CCI. Hospital volume was defined as the annual number of patients with cervical SCI at each hospital.

Clinical outcomes included in-hospital deaths and major complications (sepsis (ICD-10 codes: A40, A41), respiratory complications (pneumonia (J12-J18), postprocedural respiratory disorders (J95) or respiratory failure (J96)), pulmonary embolism (I26), gastroduodenal ulcer/bleeding (K25, K26), urinary tract infection (N10, N30, N390)).

\section{Statistical analyses}

We performed a one-to-one matching of patients in the highdose methylprednisolone group and the control group on the basis of estimated propensity scores of each patient. ${ }^{21}$ The propensity-score approach addresses selection bias that is inherent in retrospective observational studies, where outcomes can reflect a lack of comparability in treatment groups rather than the effects of treatment. This approach tries to construct a randomised experimental-like situation where treatment groups being contrasted are comparable for observing prognostic factors. Application of propensity-score matching involves estimation of the propensity score followed by matching of patients according to their estimated propensity score and comparison of outcomes in matched patients. To estimate the propensity score, we fitted a logistic regression model for the receipt of high-dose methylprednisolone treatment as a function of patient demographic and hospital factors, including age, sex, JCS score, CCI, receipt of cervical spinal surgery and hospital volume. The C-statistic for evaluating the goodness-of-fit was calculated. Each patient in the high-dose methylprednisolone group was matched with a patient in the control group with the closest estimated propensity on the logit scale within a specified range $(\leq 0.6$ of the pooled SD of estimated logits) to reduce differences between treatment groups by at least $90 \% .^{21}$

Descriptive statistics of the patient population included proportions to describe categorical variables and the median and IQR values to describe continuous variables. The $\chi^{2}$ test was used to compare categorical data and the Wilcoxon rank sum test to compare continuous variables. Fisher's exact test was used to compare in-hospital mortality and major complication rates between the high-dose methylprednisolone group and the control group. A logistic regression analysis for major in-hospital complications was performed in the propensity score-matched patients to analyse the adjusted effects of various factors, while also adjusting for clustering of patients within hospitals using a generalised estimating equation. The threshold for significance was a $\mathrm{p}$ value $<0.05$. All statistical analyses were conducted using IBM SPSS V.19.0 (IBM SPSS, Armonk, New York, USA).

\section{RESULTS}

We identified 3508 cervical SCI patients (2652 men and 856 women; mean \pm SD age, $60.8 \pm 18.7$ years) who had an emergency admission direct to the participating hospitals. Among them, we identified $824(23.4 \%)$ patients who received $\geq 5000 \mathrm{mg}$ methylprednisolone with initiation on the day of admission (high-dose methylprednisolone group). We also identified 2101 patients treated without methylprednisolone, or with $<500 \mathrm{mg}$ methylprednisolone during hospitalisation (the control group). By one-to-one propensity-score matching, 812 pairs of the high-dose methylprednisolone and control groups were selected. The C-statistic for goodness-of-fit was 0.630 in the propensity-score model, which suggested a moderately good fit.

Table 1 shows the patient demographics of the unmatched and propensity-matched groups. In the unmatched groups, 
Table 1 Patient demographics in unmatched and propensity score-matched groups

\begin{tabular}{|c|c|c|c|c|c|c|}
\hline & \multicolumn{2}{|c|}{ Unmatched group } & \multirow[b]{2}{*}{$p$ Value } & \multicolumn{2}{|c|}{ Propensity-matched group } & \multirow[b]{2}{*}{$\mathrm{p}$ Value } \\
\hline & $\begin{array}{l}\text { Control } \\
(n=2101)\end{array}$ & $\begin{array}{l}\text { High-dose methyl-prednisolone } \\
(n=824)\end{array}$ & & $\begin{array}{l}\text { Control } \\
(n=812)\end{array}$ & $\begin{array}{l}\text { High-dose methyl-prednisolone } \\
(\mathrm{n}=812)\end{array}$ & \\
\hline Sex (males, n (\%)) & $1570(74.7)$ & $645(78.3)$ & 0.044 & $650(80.0)$ & $634(78.1)$ & 0.329 \\
\hline \multicolumn{7}{|l|}{ Age (years, $n(\%)$ ) } \\
\hline$\leq 59$ & $786(37.4)$ & $318(38.6)$ & 0.022 & $292(36.0)$ & $313(38.5)$ & 0.674 \\
\hline $60-69$ & $513(24.4)$ & $219(26.6)$ & & $218(26.8)$ & $216(26.6)$ & \\
\hline $70-79$ & $456(21.7)$ & $198(24.0)$ & & $213(26.2)$ & $195(24.0)$ & \\
\hline$\geq 80$ & $346(16.5)$ & $89(10.5)$ & & $89(11.0)$ & $88(10.8)$ & \\
\hline \multicolumn{7}{|l|}{ Charlson Comorbidity Index (n (\%)) } \\
\hline 1 & $1414(67.3)$ & $456(55.3)$ & $<0.001$ & $464(57.1)$ & $456(56.2)$ & 0.638 \\
\hline 2 & $508(24.2)$ & $287(34.8)$ & & $279(34.4)$ & $276(34.0)$ & \\
\hline$\geq 3$ & $179(8.5)$ & $81(9.8)$ & & $69(8.5)$ & $80(9.9)$ & \\
\hline \multicolumn{7}{|l|}{ Japan Coma Scale at admission (n (\%)) } \\
\hline 0 (alert) & $1811(86.2)$ & $689(83.6)$ & 0.085 & $692(85.2)$ & $681(83.9)$ & 0.622 \\
\hline 1-3 (drowsy) & $200(9.5)$ & $99(12.0)$ & & 95 (11.7) & $97(11.9)$ & \\
\hline 10-30 (somnolence) & $36(1.7)$ & $20(2.4)$ & & $15(1.8)$ & $18(2.2)$ & \\
\hline $100-300$ (coma) & $54(2.6)$ & $16(1.9)$ & & $10(1.2)$ & $16(2.0)$ & \\
\hline Cervical spinal surgery & $221(10.5)$ & $189(22.9)$ & $<0.001$ & $192(23.6)$ & $178(21.9)$ & 0.408 \\
\hline $\begin{array}{l}\text { Preoperative length of stay } \\
\text { (days, median (IQR)) }\end{array}$ & $8(1-17)$ & $8(1-18)$ & 0.838 & $8(2-18)$ & $8(1-17)$ & 0.683 \\
\hline Use of tracheostomy & $55(2.6)$ & $51(6.2)$ & $<0.001$ & $38(4.7)$ & $48(5.9)$ & 0.268 \\
\hline Hospital volume (per year, median (IQR)) & $7(4-12)$ & $8(4-13)$ & 0.004 & $7(4-13)$ & $7.5(4-13)$ & 0.188 \\
\hline
\end{tabular}

patients who were male, younger, or with higher CCI were more likely to receive high-dose methylprednisolone treatment. The high-dose methylprednisolone patients were admitted to hospitals of significantly higher volume than the control group. The high-dose methylprednisolone group was significantly more likely to receive cervical spinal surgery. After propensity-score matching, patient distributions were closely balanced between the high-dose methylprednisolone and the control groups.

Table 2 shows the in-hospital mortality and major complication rates in the unmatched and propensity-matched groups. Fisher's exact test in the propensity-matched groups showed no significant difference in in-hospital mortality between the highdose methylprednisolone and control groups $(2.8 \%$ vs $3.0 \%$, $\mathrm{p}=0.884)$. There was a significant difference in gastrointestinal ulcer/bleeding $(8.4 \%$ vs $3.8 \%, p=0.001)$ between the groups. The high-dose methylprednisolone group demonstrated a significantly higher risk of overall major complications than the control group $(17.7 \%$ vs $11.8 \%, \mathrm{p}=0.001)$. Table 3 shows the results of logistic regression analysis for the occurrence of major complications. After adjustment for the measured confounders, the high-dose methylprednisolone group was significantly more likely to have major complications than the control group (OR, 1.66 ; $95 \%$ CI 1.23 to $2.24 ; \mathrm{p}=0.001$ ).

\section{DISCUSSION}

In this retrospective study using a national administrative database, patients receiving high-dose methylprednisolone after cervical SCI had a significantly higher risk of complications than those without high-dose methylprednisolone treatment. A propensity score-matched analysis revealed an increased risk of gastrointestinal ulcer/bleeding and overall major complications in the high-dose methylprednisolone group. However, high-dose methylprednisolone treatment was not associated with any increase in mortality.

\section{Strengths and weaknesses of the study}

The major strength of this study is the large size of our study sample. With a study population of 3508 patients with cervical SCI, the current analysis is the largest to examine risks associated with high-dose methylprednisolone administration. Use of the

Table 2 In-hospital mortality and major complication rates in unmatched and propensity score-matched groups

\begin{tabular}{|c|c|c|c|c|c|c|}
\hline & \multicolumn{2}{|c|}{ Unmatched group } & \multirow[b]{2}{*}{ p Value } & \multicolumn{2}{|c|}{ Propensity-matched group } & \multirow[b]{2}{*}{$p$ Value } \\
\hline & $\begin{array}{l}\text { Control } \\
(n=2101)\end{array}$ & $\begin{array}{l}\text { High-dose methylprednisolone } \\
(n=824)\end{array}$ & & $\begin{array}{l}\text { Control } \\
(n=812)\end{array}$ & $\begin{array}{l}\text { High-dose methylprednisolone } \\
(\mathrm{n}=812)\end{array}$ & \\
\hline In-hospital mortality (n (\%)) & $71(3.4)$ & $23(2.8)$ & 0.485 & $24(3.0)$ & $23(2.8)$ & 0.884 \\
\hline Major complications (n (\%)) & $191(9.1)$ & $151(18.3)$ & $<0.001$ & $96(11.8)$ & $144(17.7)$ & 0.001 \\
\hline Respiratory complications & $84(4.0)$ & $53(6.4)$ & 0.006 & $39(4.8)$ & $49(6.0)$ & 0.324 \\
\hline Urinary tract infection & $52(2.5)$ & $29(3.5)$ & 0.133 & $32(3.9)$ & $29(3.6)$ & 0.698 \\
\hline Sepsis & $16(0.8)$ & $10(1.2)$ & 0.273 & $6(0.7)$ & $10(1.2)$ & 0.330 \\
\hline Gastrointestinal ulcer/bleeding & $66(3.1)$ & $71(8.6)$ & $<0.001$ & $31(3.8)$ & $68(8.4)$ & $<0.001$ \\
\hline Pulmonary embolism & $1(0.05)$ & $4(0.5)$ & 0.024 & $1(0.1)$ & $4(0.5)$ & 0.218 \\
\hline Length of stay (median (IQR)) & $16(6-37)$ & $27(10-52)$ & $<0.001$ & $23(8-46)$ & $26(10-52)$ & $<0.001$ \\
\hline
\end{tabular}


Table 3 Logistic regression analysis of the occurrence of major complications in the propensity score-matched groups

\begin{tabular}{|c|c|c|c|}
\hline & OR & $95 \% \mathrm{Cl}$ & $\mathrm{p}$ \\
\hline \multicolumn{4}{|l|}{ Treatment } \\
\hline Control & Reference & & \\
\hline High-dose methylprednisolone & 1.66 & 1.23 to 2.24 & 0.001 \\
\hline \multicolumn{4}{|l|}{ Sex } \\
\hline Male & Reference & & \\
\hline Female & 0.57 & 0.38 to 0.86 & 0.007 \\
\hline \multicolumn{4}{|l|}{ Age } \\
\hline$\leq 59$ & Reference & & \\
\hline $60-69$ & 1.49 & 1.04 to 2.12 & 0.029 \\
\hline $70-79$ & 1.81 & 1.26 to 2.62 & 0.002 \\
\hline$\geq 80$ & 2.07 & 1.27 to 3.39 & 0.004 \\
\hline \multicolumn{4}{|l|}{ Charlson Comorbidity Index } \\
\hline 1 & Reference & & \\
\hline 2 & 1.41 & 1.04 to 1.92 & 0.027 \\
\hline$\geq 3$ & 1.95 & 1.26 to 3.02 & 0.003 \\
\hline \multicolumn{4}{|l|}{ Japan Coma Scale at admission } \\
\hline 0 (alert) & Reference & & \\
\hline 1-3 (drowsy) & 1.51 & 0.99 to 2.31 & 0.059 \\
\hline 10-30 (somnolence) & 1.75 & 0.74 to 4.09 & 0.200 \\
\hline 100-300 (coma) & 4.55 & 2.06 to 10.06 & $<0.001$ \\
\hline Cervical spinal surgery & 1.95 & 1.44 to 2.64 & $<0.001$ \\
\hline Hospital volume (per year) & 1.01 & 0.99 to 1.03 & 0.550 \\
\hline
\end{tabular}

DPC database, which covers approximately $40 \%$ of all acute hospitalisations in Japan, enabled us to conduct a nationwide investigation. In addition, the propensity score-matched analysis allowed us to evaluate the risks of high-dose methylprednisolone treatment while controlling for confounding variables, an assessment that prior studies have been unable to make.

Certain characteristics of the study subjects warrant mention. First, the mean age of the patients in this study was substantially higher than in other SCI studies, which may be explained by the rapid aging of our society. Currently, the geriatric population (those 65 years of age or older) accounts for approximately $23 \%$ of the Japanese population. Second, the surgery rate reported in this study was markedly lower compared with that of North American or European countries. The low surgery rate likely reflects differences in patient demographics and treatment strategy. In Japan, approximately $70 \%$ of patients sustain a cervical SCI without bone injury, such as fracture or dislocation (mostly elderly patients), and conservative treatment is recommended for these patients.

Our study has several limitations. First, as is common in studies using administrative data, coded diagnoses and outcomes are less well validated than prospective surveys. A degree of misclassification or under-reporting of outcome might have occurred in this study. Second, the DPC database does not provide important clinical data, such as severity of paralysis (ie, Frankel classification) at admission, patient disability at discharge, and cause of death. We could not confirm whether the administration of methylprednisolone conformed to the NASCIS protocol. Specifically, administrative databases such as the DPC database and National Inpatient Sample provide only limited information on the baseline neurological status, which is one of the most important factors that affect morbidity after SCI. It is possible that the high-dose methylprednisolone group included patients with more severe impairment than the control group, which would have created a bias toward overestimating the adverse effect of the high-dose methylprednisolone. Finally, although propensity-score adjustment is currently recognised as the best analytical approach for retrospective observational data, unmeasured confounders might have caused a hidden selection bias.

\section{Comparison with other studies}

Most published studies following the NASCIS trials indicated an increased overall complication rate after high-dose methylprednisolone treatment. ${ }^{11-15}$ Regarding specific complications, pneumonia, ${ }^{11-13} 15$ infection, ${ }^{11} 12$ and gastrointestinal bleeding ${ }^{13}$ are the most common complications reported in the literature, in patients receiving high-dose methylprednisolone. However, available evidence on the adverse effects of high-dose methylprednisolone is mixed, with substantial variation in reported incidences, and even conflicting results. There are several studies reporting lower complication rates in high-dose methylprednisolone groups. ${ }^{22} 23$ Major drawbacks of these previous studies were small sample size and lack of adjustment for confounding variables, which considerably limits the validity of their conclusion.

In the present study, we first analysed the possible adverse impact of high-dose methylprednisolone treatment in SCI patients using a large nationwide database. We then performed propensity score-matched analysis to adjust for potential confounding factors. High-dose methylprednisolone was associated with a significantly higher risk of complications $(17.7 \%$ vs $11.8 \%, \mathrm{p}=0.001$ ) than control after adjustment for confounding variables. Specifically, we found a significant increase in the occurrence of gastrointestinal ulcer/bleeding $(8.4 \%$ vs $3.8 \%$, $\mathrm{p}<0.001)$ in the high-dose methylprednisolone group.

In this study, we observed slightly lower in-hospital mortality in patients receiving high-dose methylprednisolone $(2.8 \%$ in the methylprednisolone group vs $3.0 \%$ in the control group after propensity-score matching). The impact of high-dose methylprednisolone on patient survival remains unclear. The CRASH trial, ${ }^{24}$ a randomised trial which examined the efficacy of highdose methylprednisolone in the treatment of head injury patients, was prematurely terminated because of increased 2-week mortality in the high-dose methylprednisolone group (21.1\% vs $17.9 \%)$. However, it remains to be determined whether these findings are generalisable to patients sustaining acute SCI. In fact, reported mortalities in SCI patients in the literature have been slightly more favourable in those with highdose methylprednisolone treatment, ${ }^{12} 1523$ although sample bias played a substantial role. Similarly, a meta-analysis ${ }^{18}$ of 51 randomised trials of high-dose methylprednisolone in elective and trauma surgery found reduced mortality compared with controls $(1.7 \%$ vs $2.7 \%)$, although it was not statistically significant. In our propensity score-matched analysis, no significant difference in mortality was observed between the groups in spite of a significant increase in complication rate in patients receiving high-dose methylprednisolone, which may be partly attributable to advances in intensive care and increased physician awareness of steroid-related complications.

\section{Implications for future research}

We believe that the findings of our study will provide a basis for future research to re-examine the net benefit of high-dose methylprednisolone treatment described in the NASCIS trials. The main criticism of the NASCIS trials is two-fold: (1) there was no significant difference in the primary comparison; a significant but small benefit (ie, five points in motor score) was found only after posthoc subgroup analysis; (2) there was a trend toward an increase in adverse events, including 
pneumonia, infection and gastrointestinal bleeding in patients receiving high-dose methylprednisolone. For the reasons stated above, current guidelines classify this treatment only as a therapeutic 'option', leaving the decision to adopt or avoid this treatment up to individual physicians. Despite the apparent need for a randomised study of better design with sufficient power to examine whether the beneficial effect of high-dose methylprednisolone is reproducible, no such study has been conducted mainly because of ethical and safety concerns. With a dearth of effective alternative therapeutic options, we believe that a strong case exists for a randomised placebo-controlled trial re-examining the potential benefit of high-dose methylprednisolone in patients sustaining SCI. The results of our study showed that high-dose methylprednisolone treatment was not associated with any increase in in-hospital mortality, despite a significant increase in complications, a finding that further justifies future randomised trials in carefully selected patient population. To minimise the heterogeneity of the study population, future trials should focus on patients with incomplete SCI, in whom a beneficial effect was observed in the NASCIS trial. According to an estimate by the International Campaign for Cures of Spinal Cord Injury Paralysis, ${ }^{25}$ it would require about 450 subjects with incomplete motor cervical SCI in each arm of the study to show a statistically significant difference of five American Spinal Injury Association motor points between the experimental and control groups. It would clearly require a multi-institution collaboration to carry out this project.

\section{CONCLUSION}

Despite controversies lingering for more than two decades since the publication of the NASCIS trial, risks and benefits of highdose methylprednisolone treatment remain unclear with limited high-level evidence. In this study, we focused on safety concerns of high-dose methylprednisolone treatment, and first clarified the magnitude of its adverse impact by using a large nationwide database. There was a significantly increased risk of major complications, in particular, gastrointestinal ulcer/bleeding, with high-dose methylprednisolone, but no increase in in-hospital mortality. We believe that the findings of our study provides critical information on the risks associated with high-dose methylprednisolone administration in patients with SCI, and thus, may help physicians make a more informed decision on the use of this highly controversial treatment.

Contributors $\mathrm{HC}, \mathrm{HY}, \mathrm{KT}, \mathrm{HK}$ and $\mathrm{ST}$ contributed to the conception and design of the study. $\mathrm{HH}, \mathrm{KO}, \mathrm{KF}$ contributed to the analysis, and all authors contributed to the interpretation. $\mathrm{HC}$ drafted the article; all authors revised it critically for important intellectual content and approved the final version submitted for publication. $\mathrm{HC}$ is the guarantor. All authors had full access to all of the data in the study and can take responsibility for the integrity of the data and the accuracy of the data analysis.

Funding This study was funded by a Grant-in-Aid for Research on Policy Planning and Evaluation from the Ministry of Health, Labour and Welfare, Japan (Grant number: H22-Policy-031), by a Grant-in-Aid for Research on Intractable Diseases from the Ministry of Health, Labour and Welfare, Japan (Grant number: H23-Nanchi-032), by a Grant-in-Aid for Scientific Research B (No. 22390131) from the Ministry of Education, Culture, Sports, Science and Technology, and by the Funding Program for World-Leading Innovative R\&D on Science and Technology (FIRST programme) from the Council for Science and Technology Policy, Japan (Grant number: 0301002001001). The researchers were independent from the funders. Sponsors played no role in the study design; the collection, analysis, and interpretation of data; the writing of the article; or the decision to submit it for publication.

Competing interests All authors have completed the Unified Competing Interest form at http://www.icmje.org/coi_disclosure.pdf (available on request from the corresponding author) and declare that (1) none has company support for the submitted work; (2) the authors have no relationships with companies that might have an interest in the submitted work in the previous 3 years; (3) their spouse, partners, or children have no financial relationships that may be relevant to the submitted work; and (4) the authors have no non-financial interests that may be relevant to the submitted work.

Ethics approval The Institutional Review Board at The University of Tokyo approved the study.

Provenance and peer review Not commissioned; externally peer reviewed.

Open Access This is an Open Access article distributed in accordance with the Creative Commons Attribution Non Commercial (CC BY-NC 3.0) license, which permits others to distribute, remix, adapt, build upon this work non-commercially, and license their derivative works on different terms, provided the original work is properly cited and the use is non-commercial. See: http://creativecommons.org/ licenses/by-nc/3.0/

\section{REFERENCES}

1 Bracken MB, Shepard MJ, Collins WF, et al. A randomized, controlled trial of methylprednisolone or naloxone in the treatment of acute spinal-cord injury. Results of the Second National Acute Spinal Cord Injury Study. N Eng J Med 1990;322:1405-11.

2 Bracken MB, Shepard MJ, Holford TR, et al. Administration of methylprednisolone for 24 or 48 hours or tirilazad mesylate for 48 hours in the treatment of acute spinal cord injury. Results of the Third National Acute Spinal Cord Injury Randomized Controlled Trial. National Acute Spinal Cord Injury Study. JAMA 1997;277:1597-604.

3 Sayer FT, Kronvall E, Nilsson OG. Methylprednisolone treatment in acute spinal cord injury: the myth challenged through a structured analysis of published literature. Spine J 2006;6:335-43.

4 Hurlbert RJ. The role of steroids in acute spinal cord injury: an evidence-based analysis. Spine (Phila Pa 1976) 2001:S39-46.

5 Short DJ, El Masry WS, Jones PW. High dose methylprednisolone in the management of acute spinal cord injury — a systematic review from a clinical perspective. Spinal Cord 2000;38:273-86.

6 Eck JC, Nachtigall D, Humphreys SC, et al. Questionnaire survey of spine surgeons on the use of methylprednisolone for acute spinal cord injury. Spine (Phila Pa 1976) 2006;31:E250-3.

7 Frampton AE, Eynon CA. High dose methylprednisolone in the immediate management of acute, blunt spinal cord injury: what is the current practice in emergency departments, spinal units, and neurosurgical units in the UK? Emerg Med J 2006;23:550-3.

8 Molloy S, Price M, Casey AT. Questionnaire survey of the views of the delegates at the European Cervical Spine Research Society meeting on the administration of methylprednisolone for acute traumatic spinal cord injury. Spine (Phila Pa 1976) 2001;26:E562-4.

9 Rozet I. Methylprednisolone in acute spinal cord injury: is there any other ethical choice? I Neurosurg Anesthesiol 2008;20:137-9.

10 Bracken MB. Steroids for acute spinal cord injury. Cochrane Database Syst Rev 2012;1:CD001046.

11 Ito $Y$, Sugimoto $Y$, Tomioka $M$, et al. Does high dose methylprednisolone sodium succinate really improve neurological status in patient with acute cervical cord injury?: a prospective study about neurological recovery and early complications. Spine (Phila Pa 1976) 2009;34:2121-4.

12 Suberviola B, González-Castro A, Llorca J, et al. Early complications of high-dose methylprednisolone in acute spinal cord injury patients. Injury 2008;39:748-52.

13 Matsumoto T, Tamaki T, Kawakami M, et al. Early complications of high-dose methylprednisolone sodium succinate treatment in the follow-up of acute cervical spinal cord injury. Spine (Phila Pa 1976) 2001;26:426-30.

14 Pointillart V, Petitjean ME, Wiart L, et al. Pharmacological therapy of spinal cord injury during the acute phase. Spinal Cord 2000;38:71-6.

15 Gerndt SJ, Rodriguez JL, Pawlik JW, et al. Consequences of high-dose steroid therapy for acute spinal cord injury. J Trauma 1997;42:279-84.

16 Matsuda S, Ishikawa K, Kuwabara K, et al. Development and use of the Japanese case-mix system. Eurohealth 2008;14:27-30.

17 Chikuda $\mathrm{H}$, Yasunaga $\mathrm{H}$, Horiguchi $\mathrm{H}$, et al. Mortality and morbidity in dialysis-dependent patients undergoing spinal surgery: analysis of a national administrative database in Japan. J Bone Joint Surg Am 2012;94:433-8.

18 Sauerland S, Nagelschmidt M, Mallmann P, et al. Risks and benefits of preoperative high dose methylprednisolone in surgical patients: a systematic review. Drug Saf 2000;23:449-61.

19 Ono K, Wada K, Takahara T, et al. Indications for computed tomography in patients with mild head injury. Neurol Med-Chir 2007;47:291-7.

20 Quan $H$, Sundararajan V, Halfon $P$, et al. Coding algorithms for defining comorbidities in ICD-9-CM and ICD-10 administrative data. Med Care 2005;43:1130-9.

21 Ayanian JZ, Landrum MB, Guadagnoli E, et al. Specialty of ambulatory care physicians and mortality among elderly patients after myocardial infarction. $N$ Engl J Med 2002;347:1678-86 
22 Tsutsumi S, Ueta T, Shiba K, et al. Effects of the Second National Acute Spinal Cord Injury Study of high-dose methylprednisolone therapy on acute cervical spinal cord injury-results in spinal injuries center. Spine (Phila Pa 1976) 2006;31:2992-6

23 George ER, Scholten DJ, Buechler CM, et al. Failure of methylprednisolone to improve the outcome of spinal cord injuries. Am Surg 1995;61: 659-63.
24 Roberts I, Yates D, Sandercock P, et al. Effect of intravenous corticosteroids on death within 14 days in 10008 adults with clinically significant head injury (MRC CRASH trial): randomised placebo-controlled trial. Lancet 2004;364:1321-8.

25 Fawcett JW, Curt A, Steeves JD, et al. Guidelines for the conduct of clinical trials for spinal cord injury as developed by the ICCP panel: spontaneous recovery after spinal cord injury and statistical power needed for therapeutic clinical trials. Spinal Cord 2007:45:190-205.

\section{Appendix Japan Coma Scale for grading of impaired consciousness ${ }^{19}$}

\begin{tabular}{|c|c|}
\hline Grade & Consciousness level \\
\hline 1-digit code & The patient is awake without any stimuli, and is: \\
\hline 1 & Almost fully conscious \\
\hline 2 & Unable to recognise time, place and person \\
\hline 3 & Unable to recall name or date of birth \\
\hline 2-digit code & The patient can be aroused (then reverts to previous state after cessation of stimulation): \\
\hline 20 & With loud voice or shaking of shoulders (or is almost always responsive to very simple words like yes or no, or to movements)* \\
\hline 30 & Only by repeated mechanical stimuli \\
\hline 3-digit code & The patient cannot be aroused with any forceful mechanical stimuli, and: \\
\hline 100 & Responds with movements to avoid the stimulus \\
\hline 200 & Responds with slight movements including decerebrate and decorticate posture \\
\hline 300 & Does not respond at all except for change of respiratory rhythm \\
\hline
\end{tabular}

' $R$ ' and ' $I$ ' are added to the grade to indicate restlessness and incontinence of urine and faeces, respectively: for example; 100-R and 30-RI.

${ }^{*}$ Criteria in parentheses are used in patients who cannot open their eyes for any reason. 